\title{
9 Liste des segments mis en exergue par le traducteur
}

Car, quant en deliberant d'aucune matiere, utilité ravist à luy le courage de l'omme d'un costé et honnesteté de l'autre, adonc, l'entendement de l'omme est destrait de deliberer et cela engendre en luy une sollicitude doubteuse, qui le fait estre penssif (I.9.7) ;

Car toute la louenge des vertus est en operacion (I.19.5) ;

Or, le fondement de justice est foy (I.23.1) ;

Combien que pluseurs sont tirez aux biens en ceste maniere qu'ilz oublient justice quant ilz sont cheuz en la cupidité et avarice des empires et des honneurs (I.26.1) ;

Et, pour ce, dit ce philozophe Ennius qu'il n'y a sainte societé ne foy en royaume (I.26.2) ;

Mais, toutesfoiz, il est plus juste que ilz y aillent de leur volenté (I.28.7) ;

Car il est bien difficile de prendre grant cure et sollicitude des choses d'autrui (I.30.1) ;

Car raison et equité reluist d'elle mesmes et doubter engendre congnoissance d'injure (I.30.5) ;

Souverain droict, souveraine injure (I.33.3) ;

car de toute injustice il n'en est point de si grant ne de si principalle que sembler estre bon homme (I.41.7) ;

qui dient que toutes les choses des amis doivent estre communes entr'eulx (I.51.4) ;

Car, il n'est rien plus à amer ne qui soit melx assemblé que similitude de bonnes meurs (I.56.2) ;

car ce qui est plus difficile à avoir est le plus noble (I.64.8) ;

et n'est point de temps qui doie vacquer ou estre sans justice (I.64.9) ;

car celuy ne doit point estre reputé grant homme qui est loué d'une multitude de gens ignares et non cognoessans ad ce (I.65.4) ;

Car, il n'est point convenable ne consentent à nature que ung homme soit corrumpu par cupidité, qui n'est point corompable par paour. <Et n'est point semblablement convenable à nature > que ung homme qui n'est point vaincu par labour soit vaincu par volupté (I.68.1-2) ;

car il n'est rien qui soit de si petit courage que amer les richesses (I.68.4) ; et n'est rien plus honneste ne plus magnifique que despriser les peccunes, quant on n'en a point, et, quant on en a, on les doit distribuer en benificence et en liberalité. (I.68.5) ;

mais en toutes choses que on veult faire, devant que on y commance, on y doit mectre diligente preparacion (I.73.7) ;

○ Open Access. (c) 2019 Olivier Delsaux, published by De Gruyter. (c) BY-NC-ND This work is licensed under the Creative Commons Attribution-NonCommercial-NoDerivatives 4.0 License. 
car les batailles et armes sont bien petites dehors s'il n'y a du conseil en l'ostel (I.76.27) ;

les armes donnent lieu à la chappe et le heaume donne lieu à la langue, <c'est à dire que la science vault mieulx que les armes>. (I.77.2) ;

Et pour tant on doit plus desirer la raison de discerner par science que celle de batailler par armes (I.80.1) ;

Et devons prendre la bataille en telle maniere que nous ne desirons autre chose que la paix (I.80.3) ;

Or, doncques, il appartient au fort et constant courage <de l'omme>, <ainsi comme on dit communement>, qu'il ne se trouble point es choses aspres <et qui viennent contre sa volenté> et qu'il ne soit point mis hors de son degré par les tumultes et impetuositez <de Fortune> (I.80.4) ;

Et, non pour tant, quant le temps et la necessité le requierent, on doit batailler par mains et doit on mielx amer la mort que avoir honte et estre mis en servitude de ses ennemis. (I.81.6) ;

Mais, ambicion et contenement de honneur est une chose du tout mauvaise (I.87.1) ;

Car il n'est riens plus à louer que ung grant et noble homme et n'est rien plus digne que quant ung homme est clement et doulx à appaiser (I.88.4) ;

car il est impossible que celui qui par ire veult pugnir aucun puisse garder le moyen, qui est entre trop et peu, qui plaist <beaucoup $>$ aux peripathetiques (I.89.4) ;

que de tant plus que nous suismes grans, de tant plus nous nous portions bassement (I.90.7) ;

Car ce qui est fait virilement et de grant courage semble à ung home preux estre digne et honnourable. Mais, ce que est fait au contraire lui semble estre leit et infame (I.94.9-10) ;

Car ung homme n'est pas seulement arrogant, mais est du tout dissolu qui ne retient point de extimacion de lui mesme et desprise la vertu que ung aultre sçait et voit en luy. (I.99.2) ;

Et, doncques, s'il est aucun qui soit ung peu trop adonné à plaisir et qui soit prins de checune volupté, toutesfoys, si doit il muer et dissimuler par honte l'appetit d'icelle volupté. (I.105.8) ;

Car il ne appartient point à ung homme de repugner à sa nature ou faire chose à quoy il ne puisse advenir. (I.110.4) ;

$<$ Et, pour ce, doncques, le proverbe est vroy qui dit que $>$ ung foul voit <aucunesfoiz> à ung disner telle chose que ung sage homme ne verra pas <en toute sa vie> (I.114.3) ;

Et, pour tant, qui veult bailler tout le conseil de vie à la maniere de sa nature, qui n'est point vicieuse, il doit tenir constance (I.120.6) ; 
Et aussi il n'est rien que ung vueil homme doye plus garder que de se adonner à langueur et paresse (I.123.4) ;

Et, combien que luxure soit infame en tout l'aage <de l'omme>, toutesfoiz, si est elle puante et abhominable <principallement> en ung vueil homme (I.123.5) ;

Mais, il n'est rien qui soit si convenable que garder constance en toutes les choses que on veult faire, et <semblablement> en prennant conseil (I.125.3) ; esquelz, come en pluseurs autres choses, tout homme doit tenir mediocrité (130.11) ;

Mais, en la plus grant partie de sa correction, on doit user de castigation clemente en y joignant gravité (I.137.1) ;

Car les choses qui se font par perturbation ne puent estre faictes par constance et ne sont point approuvees de ceulx qui sont presens (I.137.5) ;

$\mathrm{O}$ ancienne maison, helas, que tu es gouvernee par ung seigneur qui n'est pas semblable à celui qui te souloit gouverner, laquelle parolle peut bien estre dicte de pluseurs en ce temps present (I.139.8) ;

$<0$, Sophocles>, il ne appartient pas à ung juge seulement avoir les mains abstinentes, mais aussi fault qu'il ait les yeulx abstinens (I.144.7) ;

Mais il n'est rien plus lait que acoustumer à mentir (150.5) ;

Car la chose va bien mal quant ung homme a par peccune ce qu'il doit avoir par vertus (II.22.1) ;

Et, pour ce, doncques, il n'est point de si grant force de empire ou de domination qui puisse durer longuement quant celui qui est en celle dominacion ou empire se fait craindre (II.25.12) ;

Car ceulx qui ne sont point ornez des vertus sont tourmentez de plusieurs injures (II.39.4) ;

Car, la vraie gloire fait de grandes racines et se estent grandement, mais toutes choses fainctes descheent legierement come fleurs, tellement que une chose faincte ne peult durer longuement (II.43.3) ;

Et, semblablement, on doit tenir <celle vertus $>$ que on ne accuse jamais en jugement ung homme qui soit innoscent <du cas>, car on ne le sauroit faire sans grant peché (II.51.1) ;

$<$ Et, semblablement, quant on a fait les grandes> largicions, les rapines s'en ensuivent, car quant par trop donner homme vient à pouvreté, il est contraint de mectre les mains aux choses d'autrui et de les embler (II.54.6) ;

car, comme dit le poete Ennius, les biensfaiz qui sont mal assis sont mal faiz (II.62.4) ;

Car, nostre volenté est prés que tousjours plus encline à faire plesir à celui de qui nous actendons estre plustost remunerez et plus grandement (II.69.6) ;

Et, pour ces causes, il me semble que ung homme doit plustost colloquer ses biensfaiz envers les bons povres que envers les riches fortunez (II.71.1) ; 
<ausquelz il respondit>, j'ameroye melx, dist il, donner ma fille à ung homme qui ait faulte de peccune que à de la peccune qui ait faulte d'un homme (II.71.5) ;

Car il n'y a chose qui entretiegne si grandement les citoiens que foy, laquelle ne peult avoir force, si non qu'il soit necessité aux debteurs de poyer ce qu'ilz ont emprunté (II.84.4) ;

Et n'est chose utile qui ne soit honneste (III.11.9) ;

Et, par ce que dit est, nous voyons que ung homme qui obeist à nature ne sauroit nuyre à ung autre (III.25.4) ;

mais nous devons entendre que utilité ne peult estre avecques turpitude (III.35.3) ;

Et, pour ce, doncques, on ne doit point prendre de deliberacion des choses esquelles la deliberacion est deshonneste (III.37.3) ;

Et, semblablement, on doit oster toute son esperance et son oppinion de toute deliberacion de faire les choses sceleement et occultement (III.37.4) ;

Et, quant tu voudras donner ta sentence, tu doiz avoir en memoire de appeller Dieu en tesmoing de ce que tu diras (III.44.1) ;

Mais, une chose qui est cruelle n'est jamais utile (III.46.10) ;

mais qu'il ne face rien contre les loys escriptes ne contre les coustumes, car nous ne voullons pas seulement estre riches pour nous, mais aussi pour noz enffans, noz parens, noz prochains et noz amis et, principallement, pour la chose publique, car les copies et les facultez d'un checun citoien sont les richesses de la cité (III.63.3-5) ;

Et, pour ce, les fraudes et decepcions doivent estre ostees et aussi la fraude qui veult sembler et estre dicte prudence, mais elle en est bien loing <et y a grant difference entre fraude et prudence> (III.71.1-2) ;

Car, il n'y a point de plusgrande mauvaistié en malice que monstrer semblance de prudence où il n'y en a point (III.72.3) ;

Et, pour toute resolucion, il me semble que les heritaiges ne sont point vraiz heritaiges ne honnestes quant ilz sont acquis par malicieuses fraudes de vertus ou par simulacion et non point par verité (III.74.6) ;

$<$ Mais, certes $>$, il y a beaucoup de gens iniques et desloyaux qui regnent et y en a bien peu de bons, ce dit Actius (III.84.5) ;

ou se on veult desirer des richesses, elles ne pourront jamais estre utiles avecques infamie (III.87.3) ;

<Et se tu me demandes la cause pour quoy ilz les separent, je te respons que> c'est pour ce que nous desirons tous utilité et y mectons toute nostre pencee, et si ne le voullons point faire autrement (III.101.7) ;

Car, quant tu as fait ung serment et ta pencee conceoit que tu le doiz tenir, lors tu le doiz garder (III.107.2) ; 
Car, les anciens <qui estoient devant luy et aussi ceulx qui estoient de son temps>, disoient qu'il n'y avoit point de lyen qui lyast si fort la foy <de l'omme> comme faisoit le jurement (III.111.6) ;

Car, la servitute des vertus qui sert à volupté est inique et mauvaise (III.117.8). 